\title{
Correlation between mammographic density and volumetric fibroglandular tissue estimated on breast MR images
}

\author{
Jun Wei, ${ }^{a)}$ Heang-Ping Chan, Mark A. Helvie, Marilyn A. Roubidoux, Berkman Sahiner, \\ Lubomir M. Hadjiiski, Chuan Zhou, Sophie Paquerault, Thomas Chenevert, and \\ Mitchell M. Goodsitt \\ Department of Radiology, University of Michigan, Ann Arbor, Ann Arbor, Michigan 49109
}

(Received 18 June 2003; revised 26 November 2003; accepted for publication 21 January 2004; published 26 March 2004)

\begin{abstract}
Previous studies have found that mammographic breast density is highly correlated with breast cancer risk. Therefore, mammographic breast density may be considered as an important risk factor in studies of breast cancer treatments. In this paper, we evaluated the accuracy of using mammograms for estimating breast density by analyzing the correlation between the percent mammographic dense area and the percent glandular tissue volume as estimated from MR images. A dataset of 67 cases having MR images (coronal 3-D SPGR T1-weighted pre-contrast) and corresponding 4-view mammograms was used in this study. Mammographic breast density was estimated by an experienced radiologist and an automated image analysis tool, Mammography Density ESTimator (MDEST) developed previously in our laboratory. For the estimation of the percent volume of fibroglandular tissue in breast MR images, a semiautomatic method was developed to segment the fibroglandular tissue from each slice. The tissue volume was calculated by integration over all slices containing the breast. Interobserver variation was measured for 3 different readers. It was found that the correlation between every two of the three readers for segmentation of MR volumetric fibroglandular tissue was 0.99 . The correlations between the percent volumetric fibroglandular tissue on MR images and the percent dense area of the CC and MLO views segmented by an experienced radiologist were both 0.91 . The correlation between the percent volumetric fibroglandular tissue on MR images and the percent dense area of the CC and MLO views segmented by MDEST was 0.91 and 0.89 , respectively. The root-mean-square (rms) residual ranged from $5.4 \%$ to $6.3 \%$. The mean bias ranged from $3 \%$ to $6 \%$. The high correlation indicates that changes in mammographic density may be a useful indicator of changes in fibroglandular tissue volume in the breast. () 2004 American Association of Physicists in Medicine. [DOI: 10.1118/1.1668512]
\end{abstract}

Key words: mammography, breast density, MR images, correlation

\section{INTRODUCTION}

Studies have shown that there is a strong positive correlation between breast parenchymal density imaged on mammograms and breast cancer risk. ${ }^{1-3}$ The relative risk is estimated to be about 4 to 6 for women whose mammograms have parenchymal densities over $60 \%$ of the breast area, as compared to women with less than 5\% densities. Other cohort studies $^{4-13}$ also found that breast cancer risk in the category with the most extensive dense tissue was 1.8 to 6 times as high as that in the category with the least extensive dense tissue. Mammographic density as the risk indicator is greater than almost all other risk factors of breast cancer. ${ }^{2,14} \mathrm{Al}$ though there is no direct evidence that changes in mammographic breast densities will result in changes in breast cancer risk, the strong correlation between breast density and breast cancer risk has prompted researchers to use mammographic density for monitoring the effects of intervention as well as for studying breast cancer etiology. ${ }^{14-17}$

A number of researchers have investigated image analysis techniques to estimate breast density. ${ }^{15,18-28}$ The common approaches are to analyze the textural pattern or the percentage of mammographic densities relative to the breast area. It has been found that the texture measures were corre- lated with parenchymal density patterns but they appeared to be less sensitive measures of relative risk than the percent dense area. ${ }^{1,25,29}$ In current practice, breast density is estimated mainly by radiologists' visual judgment of the fibroglandular tissue imaged on mammograms following the Breast Imaging-Reporting and Data System (BI-RADS) lexicon. ${ }^{30,31}$ Because of the qualitative and subjective nature of visual judgment, there are large intraobserver and interobserver variations in the estimated breast density. The large variability may reduce the observed correlation between breast cancer risk and breast density. It may also reduce the sensitivity of studies using mammographic density for monitoring the effect of risk modifying treatments. We have developed an automated image analysis system, Mammographic Density ESTimator (MDEST), to assist radiologists in estimating breast density on mammograms. A computerized analysis is expected to increase the reproducibility and consistency in the estimation of mammographic density, thereby improving the accuracy of the related studies. In our previous study, we have found that the percent mammographic density segmented by MDEST agreed closely with that estimated by radiologists' interactive thresholding. ${ }^{32}$

The high correlation between breast cancer risk and breast 
density indicates that breast cancer risk may be closely related to the volume of glandular tissue in the breast. Among the modalities available for breast imaging at present, magnetic resonance (MR) imaging is likely to be the most accurate method for volumetric dense tissue estimation because fibroglandular tissue and adipose tissue can be well distinguished in MR images when a proper image acquisition technique is used. ${ }^{33}$ However, MR imaging is expensive, making it difficult to use MR imaging as a routine monitoring tool. ${ }^{33,34}$ On the other hand, a mammogram is a twodimensional (2-D) projection image of a three-dimensional (3-D) object. The area of dense tissue measured on a mammogram is not an accurate measure of the volume of fibroglandular tissue in the breast because no thickness information is used. However, mammography is a widely available low cost procedure that may be used for monitoring breast density change during preventive and interventional treatment or other studies. Women who participate in screening will also have mammograms readily available for retrospective review. Therefore, mammography will most likely be the method of choice for breast density estimation.

In this study, we investigated the correlation between the volumetric fibroglandular tissue in the breast and the projected breast dense area on mammograms by analyzing the percent volumetric fibroglandular tissue in MR breast images and the percent dense area in corresponding mammograms. Our purpose in this study is not to evaluate the usefulness of either MR fibroglandular tissue volume or mammographic density as an indicator for breast cancer risk, which have been studied by other investigators. Rather, we used the MR breast images to estimate the volumetric fibroglandular tissue in the breast and explored the reason that a change in mammographic density (2-D) can be used as an indicator of breast density change (3-D). These comparisons will provide a better understanding of their relationship, and may lead to improved methods for utilizing mammographic density as a surrogate marker for breast cancer risk.

\section{MATERIALS AND METHOD}

\section{A. Dataset}

In a previous study, gadolinium contrast enhanced MR dynamic imaging was employed to characterize malignant and benign breast lesions. A dataset was collected with IRB approval which included MR images and corresponding mammograms acquired between detection and before biopsy for a given patient. In the MR study, several series of images were acquired for each patient. Patients were scanned prone using a commercial dual phased-array breast coil. The imaging protocol included a series was the coronal 3-D T1weighted pre-contrast series (coronal sections 2-5 mm thick, 32 slices; 3-D Spoiled Gradient-Recalled Echo (SPGR); TE $=3.3 \mathrm{~ms} ; \mathrm{TR}=10 \mathrm{~ms}$, Flip $=40^{\circ}$, matrix $=256 \times 128, \mathrm{FOV}$ $=28-32 \mathrm{~cm}$ right/left, $14-16 \mathrm{~cm}$ superior/inferior, scan time $=2 \min 38 \mathrm{sec}$ ). This $3-\mathrm{D}$ SPGR sequence produces full volume coverage of both breasts with contiguous image sections. The dense parenchyma and fat tissue are well separated with this heavily T1-weighted acquisition. We used a set of 67 patients to study the correlation between the 2-D projected percentage of dense area on a mammogram and the percentage of dense tissue volume estimated from the 3-D MR images.

The mammograms consisting of the craniocaudal (CC) view and the mediolateral oblique (MLO) view of both breasts of the patient were digitized with a LUMISYS 85 laser film scanner at a pixel size of $50 \mu \mathrm{m} \times 50 \mu \mathrm{m}$. The digitizer has a gray level resolution of 12 bits and a nominal optical density (O.D.) range of 0 to 4 . For density segmentation, it is not necessary to use very high-resolution images. To reduce processing time, the full resolution mammograms were first smoothed with a $16 \times 16$ box filter and subsampled by a factor of 16 , resulting in $800 \mu \mathrm{m} \times 800 \mu \mathrm{m}$ images for this study.

\section{B. Estimation of fibroglandular tissue volume on MR images}

Since it is not our intention to routinely segment MR images for breast density estimation, we did not attempt to develop an automated method for this application. Our algorithm for segmentation of volumetric fibroglandular tissue on MR images used a semi-automatic method. The computer performed an initial segmentation. A graphical user interface (GUI) was developed to allow a user to review the segmentation of every slice and make modifications if necessary. The method consists of four steps. First, the breast boundary was detected automatically on each slice. A deformable model and manual modification were used to correct for incorrectly detected boundaries that usually occurred in slices near the chest wall where there were no well-defined breast boundaries. Because of inhomogeneity of the breast coil sensitivity, the signal intensity in the breast region was not uniform across the field of view. A background correction technique that estimated the low frequency background from the gray levels along the breast boundary was developed to reduce this systematic nonuniformity. Manual interactive thresholding of the gray level histogram in the breast region was then used to separate the fibroglandular from the fatty region. Morphological erosion was used to exclude the skin voxels along the breast boundary. Finally, the volume of fibroglandular tissue was calculated by integration over all slices containing the breast. A flow chart of our algorithm is shown in Fig. 1.

\section{Breast boundary detection}

A two-step algorithm was developed for the detection of breast boundary on each slice. First, we used a seeded pixel thresholding algorithm (SPTA) for the initial assessment of a breast boundary. Second, a 2-D active contour algorithm further refined the boundary. For slices close to the chest wall where no clear boundary can be seen, manual modification was used to outline an estimated boundary.

The SPTA determined the optimal threshold by iteratively partitioning the MR image into two parts and using the gradient value along the boundary of the partition as a guide in optimizing the threshold. First, the center of gravity was se- 


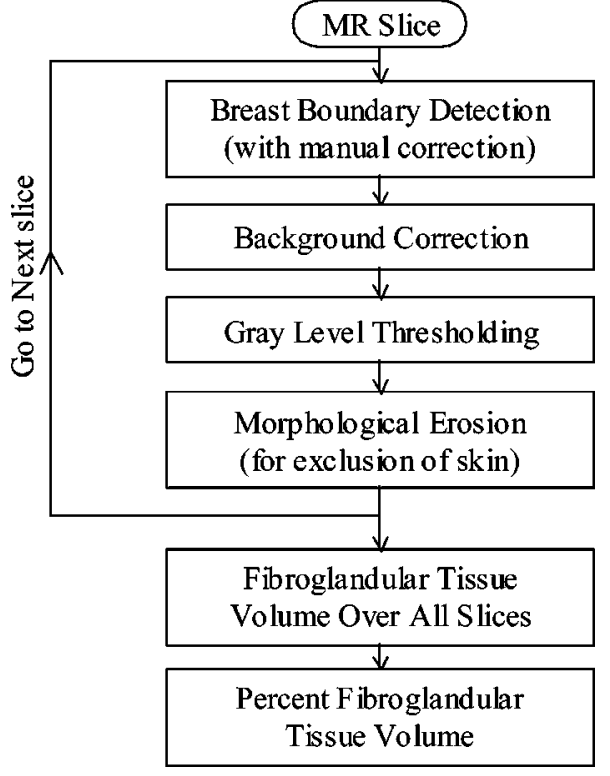

FIG. 1. The flow-chart for the segmentation of the fibroglandular tissue on MR images.

lected as the starting pixel on each slice. The gray level of the starting pixel was used as a threshold to create a binary partition of the image in which all pixels greater than the threshold were set to one and all other pixels were set to zero. Second, the gradient value of each pixel on the boundary of the binary partition was calculated by applying the Sobel filter to the original image. The gradient assessment for this particular binary partition was defined as the average gradient magnitude of these boundary pixels. The threshold value was reduced to zero in a stepwise manner. The partition for each threshold value was created and the gradient assessment for each partition was calculated as described above. The partition with the maximum gradient assessment was considered to be the initial segmentation result for the breast, and the boundary of this partition was considered to be the initial breast boundary.

After the initial segmentation, a deformable contour method was used to further refine the boundary. The movement of the boundary pixel was controlled by an energy function which consisted of internal energy and external energy. The internal energy components used in this study were the continuity and curvature of the contour, as well as the homogeneity of the segmented partition. The external energy components were the negative of the smoothed image gradient magnitude, and a balloon force that exerted pressure at a normal direction to the contour. The energy function was defined as the following:

$$
E=\sum_{c=1}^{N}\left[E_{\text {inter }}(c)+E_{\text {exert }}(c)\right]
$$

where $E_{\text {inter }}$ and $E_{\text {exert }}$ are the internal energy and the external energy, respectively, as defined in Eq. (2) and Eq. (3):

$$
E_{\text {inter }}=w_{\text {curv }} E_{\text {curv }}(c)+w_{\text {cont }} E_{\text {cont }}(c)+w_{\text {hom }} E_{\text {hom }},
$$

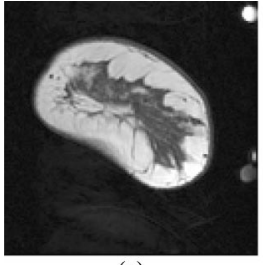

(a)

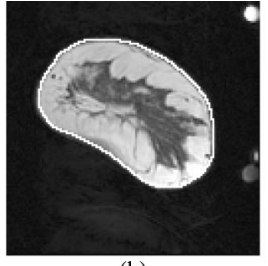

(b)

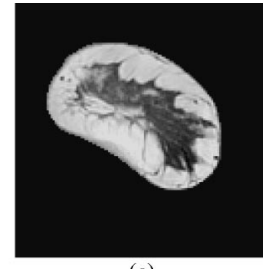

(c)
FIG. 2. An example of the first three processing blocks in Fig. 1. (a) Original MR slice; (b) automatically-detected breast boundary superimposed on the image; and (c) the background-corrected image.

$$
E_{\text {exert }}=w_{\text {grad }} E_{\text {grad }}(c)+w_{\text {bal }} E_{\text {bal }}(c),
$$

where curv, cont, grad, bal, hom denoted curvature, continuity, gradient, balloon force and homogeneity, respectively, and each energy term was associated with a weight, $w$. The detailed definition for each term can be found in the literature. ${ }^{35}$ An example of a MR slice of a breast is shown in Fig. 2(a), and the segmented boundary is shown in Fig. 2(b). Note that the two breasts of a patient were scanned together but each breast was analyzed separately.

\section{Background correction}

To reduce the nonuniformity of the MR signal intensity in the breast region, a background correction technique ${ }^{36}$ using the pixel values around the segmented breast region was employed. For a given pixel $(i, j)$ inside the breast region, the gray value of the background image was estimated as shown in Eq. (4):

$$
B(i, j)=\left[\frac{L}{d_{l}}+\frac{R}{d_{r}}+\frac{U}{d_{u}}+\frac{D}{d_{d}}\right] /\left[\frac{1}{d_{l}}+\frac{1}{d_{r}}+\frac{1}{d_{u}}+\frac{1}{d_{d}}\right],
$$

where $L, R, U$ and $D$ are the average gray values inside a breast background estimation region (BBER) centered at the left, right, upper and lower pixels on the breast boundary, respectively. A BBER was defined as the intersection of a $21 \times 21$-pixel box and the breast region. The center pixels for the left and right boxes were the intersection points between the breast boundary and a horizontal line passing through the given pixel $(i, j)$. Similarly, the upper and lower center pixels for the upper and lower boxes were the intersection points between the breast boundary and a vertical line passing through the given pixel $(i, j)$. Only the pixels that were within the intersected area between the $21 \times 21$-pixel box and the breast region were included in the definition of the BBER and the calculation of the average gray value. The contributions of the average gray levels to the background pixel $(i, j)$ were inversely weighted by their distances $d_{l}, d_{r}, d_{u}, d_{d}$ from the given pixel $(i, j)$. An example of the background corrected image is shown in Fig. 2(c).

\section{E. Segmentation of fibroglandular tissue}

We developed a GUI that allowed the user to perform a combination of manual and automatic operations to segment the breast boundary and the fibroglandular tissue on the MR 


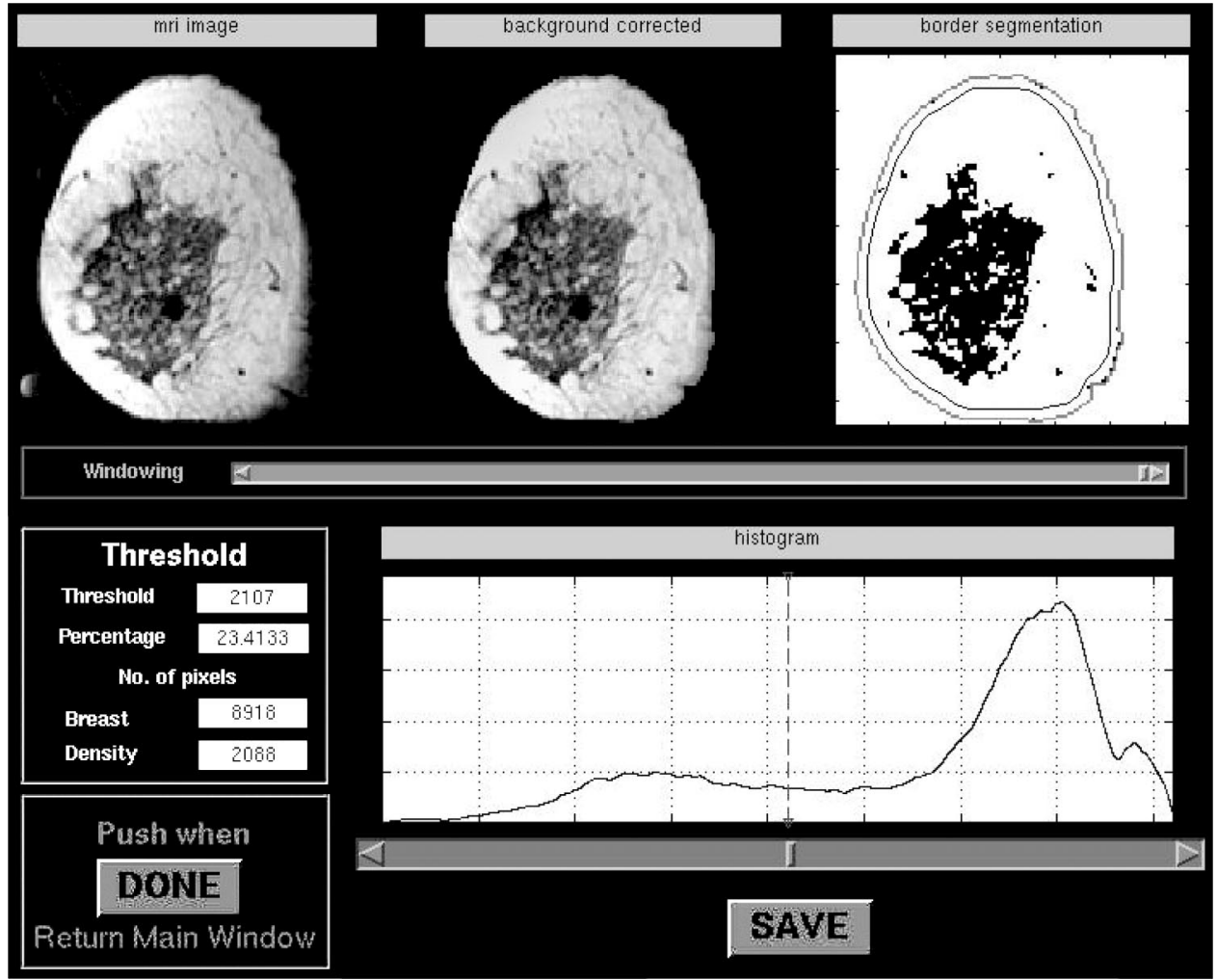

FIG. 3. The graphic user interface for the segmentation of the fibroglandular tissues on the MR slice. The upper row shows the original MR slice (left), the background-corrected image (middle) and the segmented binary image (right). The segmented image responds to the reader's adjustment of the gray level threshold (lower row) in real time so that the reader can choose the appropriate threshold by inspecting the segmented image visually. The dark area in the segmented image indicates the fibroglandular tissue and the white area indicates the adipose tissue. The inner line along the breast boundary is the boundary obtained by morphological erosion to exclude the skin voxels for calculating the fibroglandular tissue volume.

images. The first window (not shown) displayed the MR series and the corresponding mammogram of each breast to give the user an overview of the breast. The segmentation of the fibroglandular tissue on each MR slice was processed in the second window, shown in Fig. 3. The original MR slice, the corresponding background corrected image and the segmented binary image were shown in the upper part of the window. At the lower part of the window, the histogram of the voxel values in the breast region was shown. The user performed interactive thresholding on the histogram and the segmented binary image corresponding to the chosen threshold was displayed in real time in the upper part. If the breast boundary, which was automatically segmented by the computer initially, had to be corrected, the user could go to the third window and manually move the apices of the polygon outlining the boundary. The voxels contributed by the nipple were excluded. On the slices containing breast skin that had voxel values similar to those of fibroglandular tissue, a morphological erosion operation was applied to the breast boundary to exclude the skin voxels from the calculation of the fibroglandular tissue volume in the slice. The size of the structuring element could be selected interactively on the fourth window and the eroded boundary was displayed instantly for a chosen erosion operation. The user might again change the structuring element if the erosion result of the previous choice was deemed unsatisfactory. Since the eroded boundary only marked the region within which the fibroglandular voxels would be summed and would not be used for the calculation of the breast volume, as described below, it did not need to be precise as long as it excluded the skin voxels while not excluding the fibroglandular voxels.

\section{F. MR fibroglandular tissue volume}

After the fibroglandular tissue was segmented for each slice, the total number of voxels containing the fibroglandular tissue was obtained as a summation of these voxels over all slices of the breast. The total volume of the breast was obtained as the summation of the voxels enclosed by the breast boundary before morphological erosion. The ratio of these two volumes provided the percent volumetric fibroglandular tissue in the breast.

\section{G. Mammographic density segmentation}

We have previously developed an automated method for segmentation of the dense fibroglandular area on mammograms. The method, referred to as the Mammographic Density ESTimator (MDEST) was described in detail elsewhere. ${ }^{32}$ In brief, the breast boundary on the digitized mammogram is tracked. A dynamic-range compression technique reduces the gray level range of the breast area. By analyzing the shape of the gray level histogram, a rule-based classifier classifies the breast density into one of four classes. Typically, a Class I breast is almost entirely fat; it has a single narrow peak on the histogram. A Class II breast contains scattered fibroglandular densities. Its histogram has two main peaks, with the smaller peak on the right of the bigger one. A Class III breast is heterogeneously dense. Its histogram also has two peaks, but the smaller peak is on the left of the bigger one. A Class IV breast is extremely dense. Its histogram has mainly a single dominant peak, but the peak is wider compared with the peak in the Class I histogram. A second smaller peak sometimes occurs on the left of the 


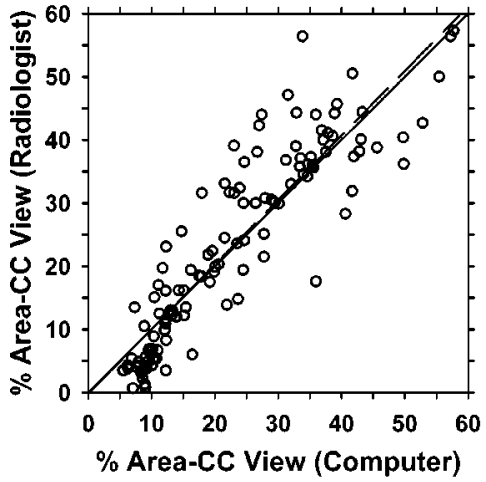

(a)

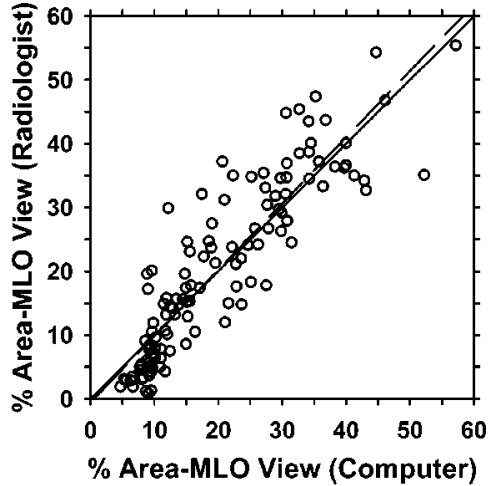

(b)
FIG. 4. A comparison of the percent mammographic density obtained from interactive thresholding by an MQSA-qualified radiologist and that estimated by our automated MDEST computer program. (a) CC view, correlation coefficient $=0.90$, rms residual $=6.7$, mean difference $=0.3$; (b) MLO view, correlation coefficient $=0.89$, rms residual $=6.1$, mean difference $=0.4$. Dashed line: linear regression of the data; solid line: diagonal. main peak. Based on the histogram shape, a threshold is automatically calculated to separate the dense and fatty pixels. The mammographic density was estimated as the percentage of fibroglandular tissue area relative to the total breast area. For MLO view mammograms, the pectoral muscle is detected and excluded from the density area or breast area calculations. In our previous work, the performance of MDEST was verified by comparison with manual segmentation by 5 breast imaging radiologists using a dataset of 260 mammograms from 65 patients that were different from the cases used in the current study. We found that the correlation between the computer-estimated percent dense area and the average segmentation by the 5 radiologists was 0.94 and 0.91, respectively, for CC and MLO views, with a mean bias of less than $2 \%$.

MDEST was applied to the mammograms of the 67 patients used in this study. The percent dense area on mammograms was estimated for the CC-view and the MLO-view mammogram of each breast separately. In addition, an MQSA-qualified radiologist also segmented the dense area by interactive thresholding for each mammogram. The correlation between the mammographic density obtained by manual and automatic segmentation is shown in Figs. 4(a) and 4(b) for the CC view and MLO view, respectively. The correlation coefficients for the CC view and MLO view were 0.90 and 0.89 , respectively. The mammographic densities estimated by automatic and manual segmentation were compared with the percent volumetric fibroglandular tissue on MR images as described below.

\section{H. Observer experiments}

We performed an experiment to evaluate the variability of the estimated \% volumetric fibroglandular tissue due to the uncertainty in the determination of the starting slice of the breast at the chest wall. The starting slice affected the estimation of the breast volume that was calculated by integrating from the starting slice to the anterior of the breast. Twenty-three MR cases from the dataset were randomly selected for this observer experiment. There were a total of 41 breasts because some cases had only one breast. For this subset of cases, each radiologist was asked to select the starting slice from the MR images for each breast. The estimated
$\%$ volumetric fibroglandular tissue calculated with all available slices was then compared to that calculated with the selected starting slice.

We also performed observer experiments to evaluate the inter-observer variations in the segmentation of fibroglandular tissue using the semi-automatic method. Two MQSAqualified radiologists performed the segmentation of the fibroglandular tissue on the MR images of the 41 breasts using the semi-automatic method implemented with the GUI. A Ph.D. researcher who was trained by these radiologists also performed the segmentation independently with the GUI.

After verifying the consistency of segmentation by these observers, the trained Ph.D. completed the segmentation of all MR cases. The correlation between percent volumetric fibroglandular tissue on MR images and percent dense area on mammograms was then examined for the entire dataset.

\section{RESULTS}

\section{A. Effect of selection of the starting slice}

Figure 5(a) shows the correlation of the \% volumetric fibroglandular tissue calculated using all available slices for the breast with that calculated using the selected starting slice by radiologist A for the 41 breasts. The correlation coefficient was 0.999. To compare the difference between their results, the mean difference and the root-mean-square (rms) residual, which is the residual from the linear least-squaresfitted line, were also calculated. The mean difference was 0.7 and the rms residual was 0.6 . The result is similar for radiologist B (not shown), with a correlation coefficient of 0.999 , a mean difference of 0.4 and a rms residual of 0.4 . The correlation between the $\%$ volumetric fibroglandular tissue calculated using the selected starting slice by radiologist $\mathrm{A}$ with that calculated using the selected starting slice by radiologist B was also very high with a correlation coefficient of 0.988 , a mean difference of 0.7 and a rms residual of 1.8 , as shown in Fig. 5(b). These comparisons indicated that the variability in the selection of the starting slice of the breasts did not have a strong influence on the \% volumetric fibroglandular tissue. We therefore used all available slices in the MR dataset for each breast in the following analyses. 


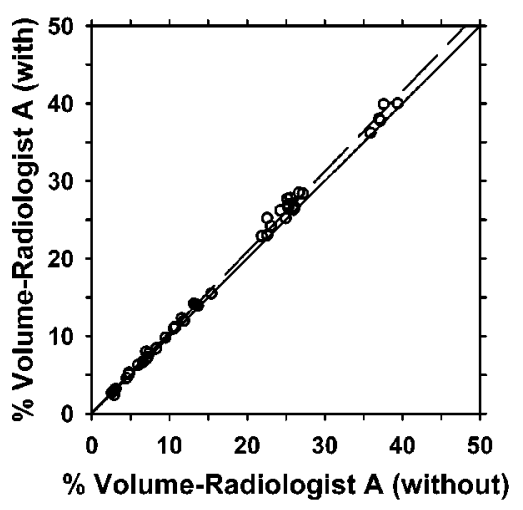

(a)

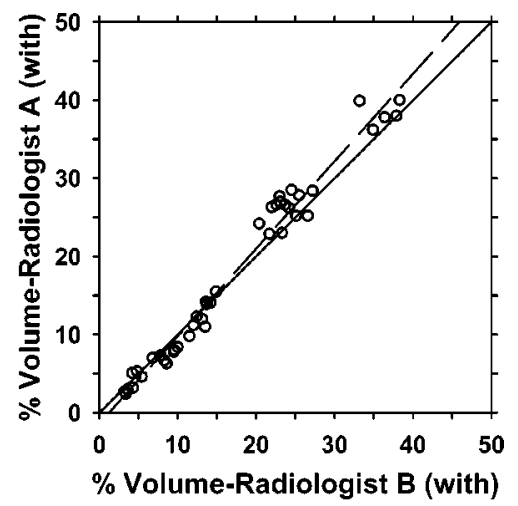

(b)
FIG. 5. (a) A comparison of the percent fibroglandular tissue volume calculated using the selected starting slice with that calculated using all available slices for radiologist $\mathrm{A}$, correlation coefficient $=0.999$. (b) A comparison of the percent fibroglandular tissue volume calculated using the selected starting slice by radiologist B with that by radiologist $\mathrm{A}$, correlation coefficient $=0.988$, Dashed line: linear regression of the data; solid line: diagonal.

\section{B. Inter-observer variation between radiologists}

Figure 6(a) shows the comparison of the percent volumetric fibroglandular tissues on MR images segmented by two radiologists for the 41 breasts. The correlation between the segmentation results of the two radiologists is 0.99 . The mean difference was found to be 0.3 and the rms residual was 1.6.

\section{Inter-observer variation between radiologists and trained Ph.D.}

Figure 6(b) shows the comparison of the percent volumetric fibroglandular tissues segmented by the trained Ph.D. against that segmented by radiologist $\mathrm{A}$. A similar result was obtained by comparing the percent volumetric tissue segmented by the trained Ph.D. and that segmented by radiologist A except that the data points were even closer to the diagonal (not shown). The correlation between the result of the trained Ph.D. and the results of both radiologists was 0.99 . The corresponding mean differences were -0.8 and -0.4 , respectively, and the rms residuals were 1.4 and 1.5, respectively.

\section{Correlation between percent volumetric fibroglandular tissue on MR images and percent mammographic density}

The percent volumetric fibroglandular tissue on MR images was compared with the percent dense area on $\mathrm{CC}$ - and
MLO-view mammograms. After verifying that the difference in segmentation between the trained Ph.D. and the radiologists was similar to the interobserver variations between the two experienced radiologists, the trained Ph.D. completed the segmentation of the entire dataset.

Figure 7 shows the comparison of the percent volumetric fibroglandular tissue on MRI and the percent mammographic density segmented by a radiologist. The percent areas on CCand MLO-view mammograms are higher than the percent volume on MR images with a mean difference of $5.7 \%$ and $3.0 \%$, respectively.

Figure 8 shows the comparison of the percent volumetric fibroglandular tissue on MRI and the percent mammographic density segmented by MDEST. The percent areas on CCand MLO-view mammograms segmented by the computer are higher than the percent volume on MR images with a mean difference of $5.3 \%$ and $2.6 \%$, respectively.

The correlation coefficients, the mean differences and the rms residuals between the percent volumetric fibroglandular tissue on MR images and percent dense area on mammograms are compared in Table. I. The correlation between the percent volume on MR images and percent area on mammograms of the fibroglandular breast tissue is high, ranging from 0.89 to 0.91 . Although it is not expected that the values of percent volume agree with the values of percent area, their mean differences range only from $3 \%$ to $6 \%$ and the rms residual range from 5.4 to 6.3 .

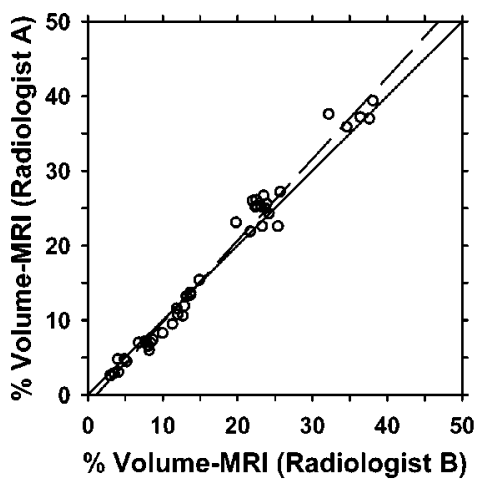

(a)

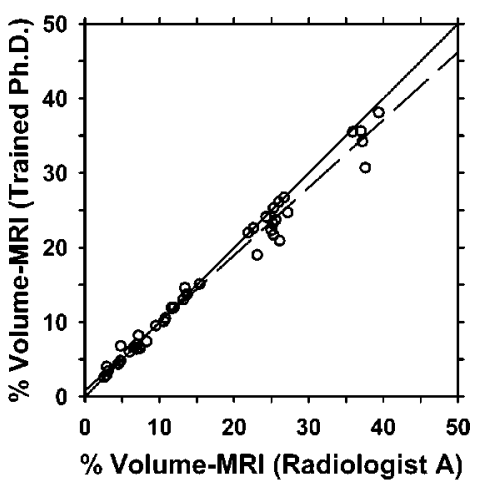

(b)
FIG. 6. A comparison of the segmentation of fibroglandular tissue from MR images between two observers: (a) two experienced MQSA-qualified radiologists, correlation coefficient $=0.99$. (b) The trained Ph.D. and Radiologist A, correlation coefficient $=0.99$. The correlation between the trained Ph.D. and Radiologist B is also 0.99 but the data points were very close to the diagonal and is not shown. The \% volumetric fibroglandular tissue was calculated using all available slices. Dashed line: linear regression of the data; solid line: diagonal. 


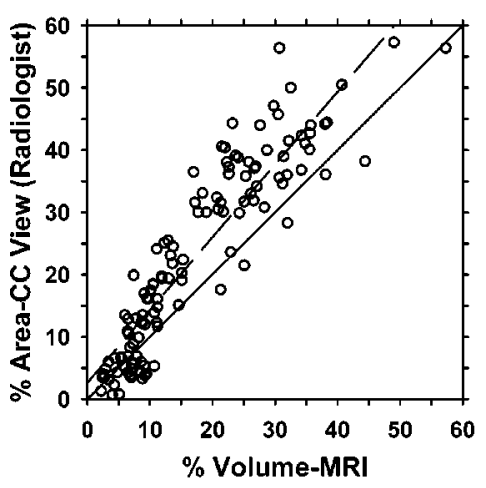

(a)

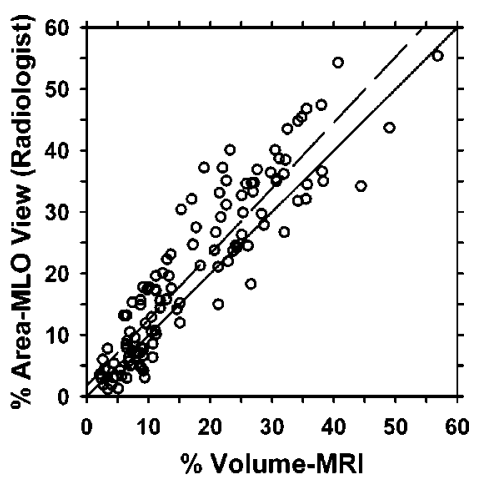

(b)
FIG. 7. A comparison of the percent fibroglandular tissue volume on MR images and the percent dense area on mammograms segmented by an experienced radiologist. (a) CC view, correlation coefficient $=0.91$; (b) MLO view, correlation coefficient $=0.91$. Dashed line: linear regression of the data; solid line: diagonal.

\section{DISCUSSION}

Our purpose in this paper was to investigate the relationship between the percent dense area on mammogram and the percent fibroglandular tissue volume on MR image. We found a direct correlation between mammographic density and MR volumetric density (Fig. 7 and Fig. 8). The correlation coefficients between the percent area on a mammogram and the percent volume on MR images are high at 0.89 and 0.91 . These results are more promising than those found in previous studies that attempted to correlate percent dense area on mammograms with MR information. Graham et al. ${ }^{33}$ investigated the relationship between percent density (projected dense area) on mammogram and two objective MR parameters of breast tissue, relative water content and mean $\mathrm{T} 2$ relaxation. Their results with 45 cases showed a positive correlation between percent density and relative water content (Pearson correlation coefficient $=0.79$ ) and a negative correlation between percent density and mean T2 value (Pearson correlation coefficient $=-0.61$ ). Another study by Lee et al $^{34}$ analyzed fatty and fibroglandular tissue in different age groups to compare $\mathrm{x}$-ray mammography with $\mathrm{T} 1-$ weighted MR images. Their study with 40 cases indicated that the correlation between the two techniques is 0.63 when the fat content was more than $45 \%$. However, the correlation coefficient decreased to 0.34 when their analysis included only dense breasts.

It may be noted that although MR imaging is currently the most accurate method for estimating the volumetric fibro- glandular tissue in the breast, it is still not the ideal tool. Fibrous tissue and glandular tissue are not well separated with current MR imaging techniques. Since the amount of glandular tissue in the breast is the important factor relating to breast cancer risk, further studies are warranted for differentiating the glandular and the fibrous components of the imaged volume. The correlation between the percent glandular tissue volume and percent projected dense area on a mammogram will be a more reliable indicator of the usefulness of mammographic density analysis.

The density on mammograms is a 2-D projected area of the fibroglandular tissues. The percent dense area is not expected to be equal in value to the percent volume. The mean differences between the percent volume and the percent area on CC- and MLO-views, as determined by the radiologist's interactive segmentation, are 5.7 and 3.0, respectively (Table I), with the percent dense area values being higher. We also investigated the rms residual between the percent volume and the percent area when the relationship between them was assumed to be linear. The rms residual between the percent volume and the percent area on CC- and MLO-views are 6.3 and 5.6, respectively (Table I), relative to the straight line obtained from linear least squares fits to the data. One possible factor that may contribute to a higher value of percent dense area on mammograms than the percent volume value on MR images is that the tissue volume imaged by the two modalities is somewhat different. The MR images include more tissue near the chest wall, which is mainly retroglan-

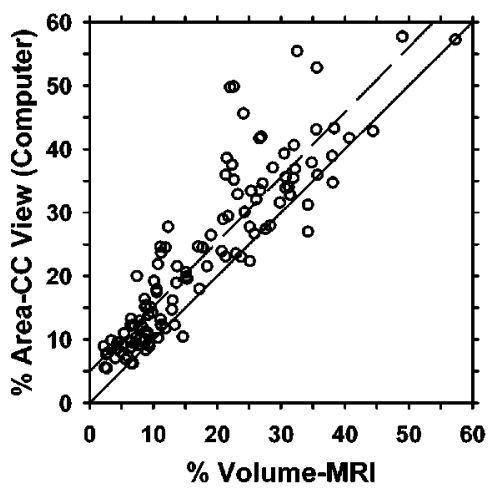

(a)

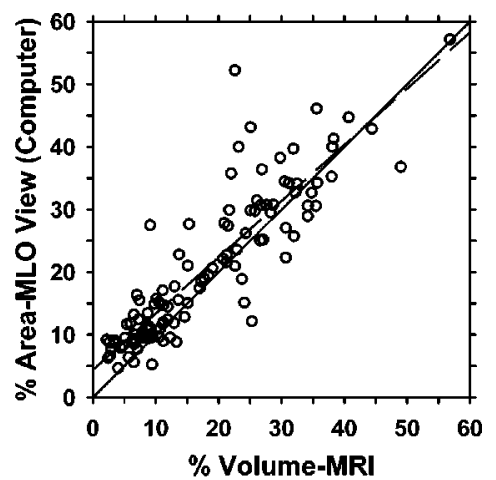

(b)
FIG. 8. A comparison of the percent volume on MR images and the percent area on mammogram segmented by our automated MDEST computer program. (a) CC view, correlation coefficient $=0.91$; (b) MLO view, correlation coefficient $=0.89$. Dashed line: linear regression of the data; solid line: diagonal. 
TABLE I. Statistic analysis of the relationship between percent fibroglandular tissue volume on breast MR images and percent dense area on mammograms segmented by radiologist and MDEST.

\begin{tabular}{lcclccc}
\hline \hline & \multicolumn{2}{c}{ Radiologist } & & \multicolumn{2}{c}{ Computer $($ MDEST) } \\
\cline { 2 - 3 } \cline { 6 - 6 } \cline { 5 - 6 } & $\begin{array}{c}\text { CC vs } \\
\text { MRI }\end{array}$ & $\begin{array}{c}\text { MLO vs } \\
\text { MRI }\end{array}$ & & $\begin{array}{c}\text { CC vs } \\
\text { MRI }\end{array}$ & $\begin{array}{c}\text { MLO vs } \\
\text { MRI }\end{array}$ \\
\hline Correl. coeff. & 0.91 & 0.91 & & 0.91 & 0.89 \\
rms residual & 6.3 & 5.6 & & 5.8 & 5.4 \\
Mean diff. & 5.7 & 3.0 & & 5.3 & 2.6 \\
\hline \hline
\end{tabular}

dular adipose tissue, than a mammogram does, thus reducing the percentage of fibroglandular tissue volume. The reduction in the percent volume values, however, is relatively small, as found in our study evaluating the effects of selecting starting slices for volume calculation (Fig. 5). The main difference may therefore be attributed to the geometric relationship between the volume and the projected 2-D area, explained later.

Geometrically, we do not expect the relationship between volume and its projected 2-D area to be linear. In a hypothetical situation such that the dense tissue volume is a sphere (volume $=4 / 3 \pi r^{3}$ ) enclosed inside a concentric spherical shell of fatty tissue volume, the percent projected 2-D area $\left(\operatorname{area}=\pi \mathrm{r}^{2}\right)$ of the inner sphere relative to the outer sphere is equal to the percent volume to the power of $2 / 3$. The relationship between the percent area and the percent volume is therefore not linear, and the percent area is larger in value than the percent volume for any ratio of radii between the two spheres. In general, the compressed breast and the dense tissue are not spherical. To investigate the empirical relationship between the percent area and the percent volume in the nonlinear situation, we applied least squares fits in several polynomial models to the data points in Fig. 7. The results are shown in Table II and Fig. 9. A comparison of Table I and Table II indicates that the $Y$ $=k x^{2 / 3}$ model $(x=$ percent fibroglandular tissue volume, $Y$ $=$ percent mammographic dense area) resulted in slightly larger rms residuals than the linear model. The model $Y$ $=k x^{m}$ with $m$ equal to 0.83 and 0.86 , respectively, for CCand MLO-views slightly reduced the rms residuals. The best fit was obtained from the model $Y=k_{1} x^{m}+k_{2}$. However, the
TABLE II. An analysis of the relationship between percent fibroglandular tissue volume $(x)$ on breast MR images and percent dense area $(Y)$ on mammograms segmented by radiologist using three mathematical models. $m, k, k_{1}$ and $k_{2}$ are constants determined by least squares curve fitting.

\begin{tabular}{ccccc}
\hline \hline Mathematical model & $Y=k x^{2 / 3}$ & $Y=k x^{m}$ & $Y=k_{1} x^{m}+k_{2}$ \\
\hline CC & Least squares Fit & $Y=0.82 x^{2 / 3}$ & $Y=1.03 x^{0.83}$ & $Y=1.02 x^{0.48}-0.19$ \\
vs & rms residual & 6.5 & 6.0 & 5.6 \\
MRI & Coefficient of & 0.82 & 0.85 & 0.87 \\
& determination & & & \\
MLO & Least squares Fit & $Y=0.73 x^{2 / 3}$ & $Y=0.96 x^{0.86}$ & $Y=0.90 x^{0.60}-0.09$ \\
vs & rms residual & 6.0 & 5.5 & 5.3 \\
MRI & Coefficient of & 0.80 & 0.84 & 0.85 \\
& determination & & & \\
\hline \hline
\end{tabular}

situation that the percent projected area was negative when the percent volume was zero would not occur physically. Note that if the model was fitted to the percent area data segmented by MDEST (Fig. 8), the $k_{2}$ values would become positive, indicating that the nonzero $k_{2}$ values are likely caused by segmentation biases.

Overall, these models demonstrate that there is no simple mathematical relationship between the percent volume and the percent projected area but the values for the exponents appeared to be in a reasonable range. The relationship between the percent volumes of two 3-D objects, one within another, and their percent projected 2-D area depends on their shapes. For example, the closer the two volumes are to concentric cylinders of the same height, the closer the exponent is to unity. The spread of the data points can therefore be attributed to the various irregular shapes of the fibroglandular tissue in the breasts, the changes in the shapes of the fatty and fibroglandular tissue due to compression, as well as the uncertainties in the segmentation of both the mammograms and the MR images. Although the spread of the data points in the correlation plots is large, one can expect that when the mammographic density of a given patient is monitored over time, the variations in the projected dense area due to the geometric factors, described above, will actually be much less than that observed from the scatter plots among a large number of patients. In other words, the uncertainty in the estimated percent density from the serial mammograms of a given patient should be much less than those shown in

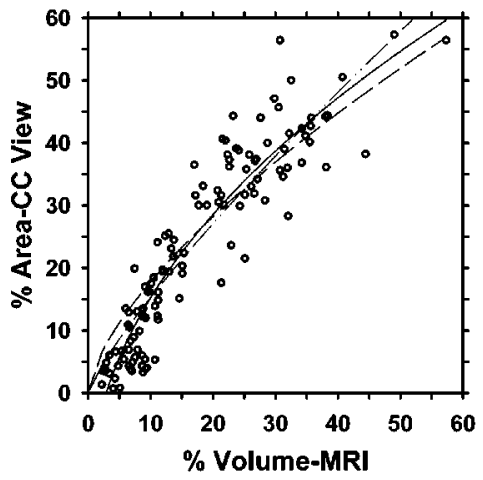

(a)

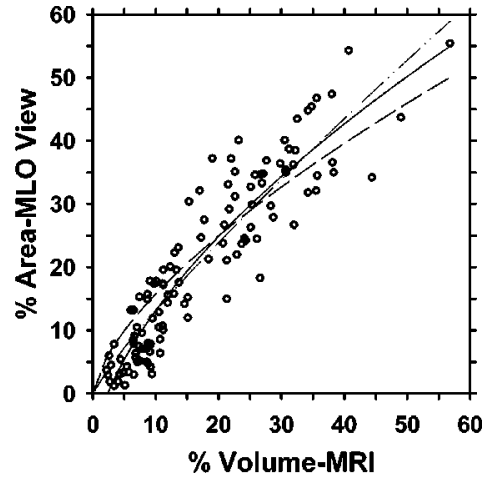

(b)
FIG. 9. Nonlinear fitting of the relationship between the percent volume and the percent area segmented by a radiologist with the least squares method. (a) $\mathrm{CC}$ view, (b) MLO view. Dashed line: $y=k x^{2 / 3}$; dashed-dotteddotted line: $y=k x^{m}$; solid line: $y=k_{1} x^{m}+k_{2}$. The fitted parameters of the models, $m, k, k_{1}$ and $k_{2}$, are shown in Table II. 
Fig. 7. The strong correlation observed between the percent dense area on mammograms and the percent volumetric fibroglandular tissue on MR images therefore indicates that a change in mammographic density can be a useful indicator of a change in percent fibroglandular tissue volume in the breast.

Recently, some researchers attempted to estimate the thickness of the fibroglandular tissue in local regions of the mammograms from the projected density. ${ }^{37}$ This approach is expected to provide a more accurate estimation of the fibroglandular tissue volume if the true thicknesses of the fibroglandular tissue and fatty tissue can be determined at various locations of the projected breast region. The volume of the fibroglandular tissue can then be summed over the pixels in the breast region and the percent volume calculated. However, to obtain accurate measurements, this approach requires the knowledge of the sensitometric curve for the screen-film mammogram at the imaging facility (or use of a digital detector with linear response) and other physical parameters such as the scatter fraction, the beam quality and beam hardening, in addition to the compressed breast thickness and the breast shape profile at the periphery. Some of the requirements may be circumvented by using a look-up table predetermined with a phantom calibration. Other factors may have to be approximated or ignored, or require further corrections by imaging each mammogram with a calibration phantom placed adjacent to the breast. This method is still being developed and the accuracy of estimating the thickness of the local fibroglandular tissue from a mammogram is yet to be determined. To our knowledge, no study to date has demonstrated that fibroglandular tissue volume estimated from mammograms has a higher correlation with the percent volumetric fibroglandular tissue volume estimated from MR images or other volumetric methods than we found in our current study. Furthermore, even if the local fibroglandular tissue thickness on mammograms can be measured in a laboratory or in an academic center using elaborate calibration schemes, it is doubtful that these methods can be translated into routine clinical measurement in mammography clinics. Its use may then be limited to controlled clinical trials. An estimation of the percent dense area projected on mammograms is likely a more practical approach for breast density assessment. The high correlation between the percent dense area and the percent fibroglandular tissue volume on MR images as demonstrated in the current study further supports the validity of this approach.

\section{CONCLUSION}

In this study, we investigated the correlation between the percent mammographic dense area and the percent volumetric fibroglandular tissue as measured on MR images. A semiautomatic method was developed for segmentation of the MR images and a fully automated computerized method, MDEST, was used to segment the mammograms. The performance of MDEST on the set of mammograms used in this study was verified with an experienced radiologist's manual segmentation. The inter-observer variability in segmentation of MR images was found to be small with correlation coefficients of 0.99. The correlation between the percent volume on MR images and percent area segmented by a radiologist for either CC- view or MLO-view is 0.91 . The correlation between percent volume and percent area estimated by MDEST is 0.91 and 0.89 , respectively, for CC and MLO views. Mammographic density is thus highly correlated with the percent volumetric fibroglandular tissue in the breast. The high correlation indicates that changes in mammographic density may be a useful indicator of changes in fibroglandular tissue volume in the breast. Our computerized image analysis tool, MDEST, can provide a consistent and reproducible estimation of percent dense area on routine clinical mammograms. The automated image analysis tool may improve the sensitivity of quantifying mammographic density changes, thereby contributing to the understanding of the relationship of mammographic density to breast cancer risk, detection, and prognosis, and the prevention and treatment of breast cancer.

\section{ACKNOWLEDGMENTS}

This work is supported by U.S. Army Medical Research and Materiel Command Grants No. DAMD 17-01-1-0326, No. DAMD 17-02-1-0214, and No. DAMD 17-99-1-9294. The content of this paper does not necessarily reflect the position of the government and no official endorsement of any equipment and product of any companies mentioned should be inferred.

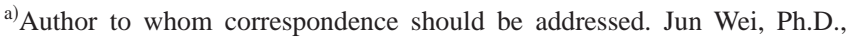
Department of Radiology, University of Michigan, CGC B2103, 1500 E. Medical Center Drive, Ann Arbor, Michigan 48109. Phone: 734-6478553; fax: 734-615-5513; electronic mail: jvwei@umich.edu

${ }^{1}$ A. F. Saftlas, R. N. Hoover, L. A. Brinton, M. Szklo, D. R. Olson, M. Salane, and J. N. Wolfe, "Mammographic densities and risk of breast cancer," Cancer (N.Y.) 67, 2833-2838 (1991).

${ }^{2}$ N. F. Boyd, G. A. Lockwood, J. W. Byng, D. L. Tritchler, and M. J. Yaffe, "Mammographic densities and breast cancer risk," Cancer Epidemiology Biomarkers \& Prevention 7, 1133-1144 (1998).

${ }^{3}$ C. M. Vachon, C. C. Kuni, K. Anderson, V. E. Anderson, and T. A. Sellers, "Association of mammographically defined percent breast density with epidemiologic risk factors for breast cancer (United States)," Cancer Causes \& Control 11, 653-662 (2000).

${ }^{4}$ P. M. Krook, "Mammographic parenchymal patterns as risk indicators for incident cancer in a screening program: an extended analysis," Am. J. Roentgenolo. 131, 1031-1035 (1978).

${ }^{5}$ R. L. Egan and R. C. Mosteller, "Breast cancer mammography patterns," Cancer (N.Y.) 40, 2087-2090 (1977).

${ }^{6}$ B. Threatt, J. M. Norbeck, N. S. Ullman, R. Kummer, and P. Roselle, "Association between mammographic parenchymal pattern classification and incidence of breast cancer," Cancer (N.Y.) 45, 2550-2556 (1980).

${ }^{7}$ M. Moskowitz, P. Gartside, and C. Mclaughlin, "Mammographic patterns as markers for high-risk benign breast disease and incident cancers," Radiology 134, 293-295 (1980).

${ }^{8}$ I. Witt, H. S. Hansen, and S. Brunner, "The risk of developing breast cancer in relation to mammography findings," Eur. Radiol. 4, 65-67 (1984).

${ }^{9} \mathrm{~S}$. Ciatto and M. Zappa, "A prospective study of the value of mammographic pattern as indicators of breast cancer risk in a screening experience," Eur. Radiol. 17, 122-125 (1993).

${ }^{10}$ E. Thurfjell, C. C. Hsieh, L. Lipworth, A. Ekbom, H. O. Adami, and D. Trichopoulos, "Breast size and mammographic pattern in relation to breast cancer risk," Eur. J. Cancer Prevention 5, 37-41 (1996).

${ }^{11}$ I. Kato, C. Beinart, A. Bleich, S. Su, M. Kim, and P. G. Toniolo, "A nested case-control study of mammographic patterns, breast volume and 
breast cancer (New York City, NY, United States)," Cancer Causes \& Control 6, 431-438 (1995).

${ }^{12}$ E. Sala, R. Warren, J. McCann, S. Duffy, N. Day, and R. Luben, "Mammographic parenchymal patterns and mode of detection: implications for the breast screening programme," J. Medical Screening 5, 207-212 (1998).

${ }^{13}$ T. M. Salminen, I. E. Saarenmaa, M. M. Heikkila, and M. Hakama, "Is a dense mammographic parenchymal pattern a contraindication to hormonal replacement therapy?," Acta Oncol. 39, 969-972 (2000).

${ }^{14}$ C. Byrne, C. Schairer, J. N. Wolfe, N. Parekh, M. Salane, L. A. Brinton, R. Hoover, and R. Haile, "Mammographic features and breast cancer risk: Effects with time, age, and menopause status," J. Natl. Cancer Inst. 87, 1622-1629 (1995).

${ }^{15}$ N. F. Boyd, C. Greenberg, G. Lockwood, L. Little, L. Martin, J. Byng, Y. Martin, and D. Tritchler, "Effects at two years of a low-fat, highcarbohydrate diet on radiologic features of the breast: Results from a randomized trial," J. Natl. Cancer Inst. 89, 466-467 (1997).

${ }^{16}$ D. V. Spicer, G. Ursin, Y. R. Parisky, J. G. Pearce, D. Shoupe, A. Pike, and M. C. Pike, "Changes in mammographic densities induced by a hormonal contraceptive designed to reduce breast cancer risk," J. Natl. Cancer Inst. 86, 431-436 (1994)

${ }^{17}$ J. Brisson, R. Verreault, A. S. Morrison, D. Tennina, and F. Meyer, "Diet, mammographic features of breast tissue, and breast cancer risk," Am. J. Epidemiol. 130, 14-24 (1989).

${ }^{18} \mathrm{~J}$. N. Wolfe, "Mammography: Ducts as a sole indicator of breast carcinoma," Radiology 89, 206-210 (1967).

${ }^{19} \mathrm{~J}$. N. Wolfe, "The prominent duct pattern as an indicator of cancer risk," Oncology 23, 149-158 (1969).

${ }^{20} \mathrm{~J}$. N. Wolfe, "Breast patterns as an index of risk for developing breast cancer," Am. J. Roentgenol. 126, 1130-1139 (1976).

${ }^{21}$ J. N. Wolfe, "Risk for breast cancer development determined by mammographic parenchymal pattern," Cancer (N.Y.) 37, 2486-2492 (1976).

${ }^{22}$ I. E. Magnin, F. Cluzeau, C. L. Odet, and A. Bremond, "Mammographic texture analysis: An evaluation of risk for developing breast cancer," Opt. Eng. 25, 780-784 (1986).

${ }^{23}$ J. W. Byng, N. F. Boyd, E. Fishell, R. A. Jong, and M. J. Yaffe, "Automated analysis of mammographic densities," Phys. Med. Biol. 41, 909923 (1996).

${ }^{24}$ J. W. Byng, N. F. Boyd, E. Fishell, R. A. Jong, and M. J. Yaffe, "The quantitative-analysis of mammographic densities," Phys. Med. Biol. 39, 1629-1638 (1994).

${ }^{25}$ M. J. Yaffe, N. F. Boyd, J. W. Byng, R. A. Jong, R. Fishell, G. A. Lockwood, L. E. Little, and D. L. Tritchler, "Breast cancer risk and measured mammographic density," Eur. J. Cancer Prevention 7, S47S55 (1998).
${ }^{26}$ Z. Huo, M. L. Giger, D. E. Wolverton, and W. Zhong, "Computerized analysis of mammographic parenchymal patterns for breast cancer risk assessment: Feature selection," Med. Phys. 27, 4-12 (2000).

${ }^{27}$ J. J. Heine and R. P. Velthuizen, "A statistical methodology for mammographic density detection,” Med. Phys. 27, 2644-2651 (2000).

${ }^{28}$ J. M. Boone, K. K. Lindfors, C. S. Veatty, and J. A. Seibert, “A breast density index for digital mammograms based on radiologists' ranking," J. Digit Imaging 11, 101-115 (1998).

${ }^{29}$ J. N. Wolfe, A. F. Saftlas, and M. Salene, "Evaluation of mammographic densities: A case-control study," Am. J. Roentgenol., Radium Ther. Nucl. Med. 148, 1087-1092 (1987).

${ }^{30}$ American College of Radiology. Breast Imaging-Reporting and Data System (BI-RADS), 3rd ed. (American College of Radiology, Reston, VA, 1998).

${ }^{31}$ E. White, P. Velentgas, M. T. Mandelson, C. D. Lehman, J. G. Elmore, P. Porter, Y. Yasui, and S. H. Taplin, "Variation in mammographic breast density by time in menstrual cycle among women aged 40-49 years," J. Natl. Cancer Inst. 90, 906-910 (1998).

${ }^{32}$ C. Zhou, H. P. Chan, N. Petrick, M. A. Helvie, M. M. Goodsitt, B. Sahiner, and L. M. Hadjiiski, "Computerized image analysis: Estimation of breast density on mammograms," Med. Phys. 28, 1056-1069 (2001).

${ }^{33}$ S. J. Graham, M. J. Bronskill, J. W. Byng, M. J. Yaffe, and N. F. Boyd, "Quantitative correlation of breast tissue parameters using magnetic resonance and X-ray mammography," Br. J. Cancer 73, 162-168 (1996).

${ }^{34}$ N. A. Lee, H. Rusinek, J. Weinreb, R. Chandra, H. Toth, C. Singer, and G. Newstead, "Fatty and fibroglandular tissue volumes in the breasts of women 20-83 years old: comparison of x-ray mammography and computer-assisted MR imaging," Am. J. Roentgenol. 168, 501-506 (1997).

${ }^{35}$ B. Sahiner, N. Petrick, H. P. Chan, L. M. Hadjiiski, C. Paramagul, M. A. Helvie, and M. N. Gurcan, "Computer-aided characterization of mammographic masses: Accuracy of mass segmentation and its effects on characterization," IEEE Trans. Med. Imaging 20, 1275-1284 (2001).

${ }^{36}$ B. Sahiner, H. P. Chan, N. Petrick, D. Wei, M. A. Helvie, D. D. Adler, and M. M. Goodsitt, "Classification of mass and normal breast tissue: A convolution neural network classifier with spatial domain and texture images," IEEE Trans. Med. Imaging 15, 598-610 (1996).

${ }^{37}$ O. Pawluczyk, B. J. Augustine, M. J. Yaffe, D. Rico, J. Yang, G. E. Mawdsley, and N. F. Boyd, "A volumetric method for estimation of breast density on digitized screen-film mammograms," Med. Phys. 30, 352-364 (2003). 